\title{
Edaravone attenuates experimental asthma in mice through induction of HO-1 and the Keap1/Nrf2 pathway
}

\author{
YUTING PAN ${ }^{1}$, WEIHAO LI ${ }^{2}$, YING FENG $^{2}$, JING XU $^{2}$ and HUIFANG CAO ${ }^{2}$ \\ ${ }^{1}$ Department of Respiratory Medicine, Shanghai Medical College of Fudan University, Xuhui, Shanghai 200032; \\ ${ }^{2}$ Department of Respiratory Diseases, Jing'an District Centre Hospital of Fudan University, Shanghai 200040, P.R. China
}

Received December 21, 2018; Accepted September 4, 2019

DOI: $10.3892 /$ etm.2019.8351

\begin{abstract}
Asthma is a chronic disease that threatens public health worldwide. Multiple studies have shown that oxidative stress plays an important role in the pathogenesis of asthma. Edaravone (Eda), a free radical scavenger, has been found to have a protective effect against lung injury due to its ability to eliminate reactive oxygen species. The present study aimed to investigate the effect of Eda on asthma and the mechanism underlying its actions. An experimental asthma model was induced in mice, before they were treated with different doses of Eda. Measurements of airway responsiveness to methacholine (Mch), cell counts and cytokine levels in bronchoalveolar lavage fluid (BALF) and of the oxidative products and antioxidant enzymes in lung tissue were taken in these asthma model mice and compared with control mice. Protein levels of kelch-like ECH-associated protein-1 (Keap1)/nuclear factor erythroid 2-related factor 2 (Nrf2) and hemeoxygenase-1 (HO-1) were determined in the lung tissue of normal mice and Nrf2 and HO-1-deficient mice subject to the asthma model to investigate the mechanisms underlying Eda action. The results indicated that Eda effectively reduced airway responsiveness to Mch. The total number of cells and the numbers of eosinophils, lymphocytes and neutrophils in BALF of asthma model mice were also significantly reduced by Eda treatment when compared with normal saline treatment. Eda treatment significantly alleviated perivascular edema, peribronchial inflammation and macrophage infiltration in the alveolar space and decreased the levels of inflammatory cytokines released in BALF compared with control. Eda also significantly reduced the levels of oxidative stress markers in BALF and restored the levels of antioxidative enzyme, superoxide dismutase, when compared with control. The Keap1/Nrf2 ratio was significantly decreased with Eda compared with control due to an
\end{abstract}

Correspondence to: Dr Huifang Cao, Department of Respiratory Diseases, Jing'an District Centre Hospital of Fudan University, 259 Xikang Road, Jing'an, Shanghai 200040, P.R. China

E-mail: hfcao999@yeah.net

Key words: edaravone, asthma, inflammation, antioxidative, nuclear factor erythroid-2 related factor 2, heme oxygenase 1 increase in Nrf2 and a decrease in Keap1 expression. HO-1 expression was increased by Eda. The airway responsiveness of $\mathrm{Nrf}^{-/-}$mice or HO-1 ${ }^{-/}$mice to Mch was significantly higher compared with normal mice treated with Eda. Taken together, the results of the present study show that Eda exerts anti-inflammatory and antioxidative effects, which suggests a potential use for Eda in reduction of asthma severity. The activated Keap1/Nrf2 pathway and HO-1 may be involved in the anti-asthmatic effect of Eda.

\section{Introduction}

Allergic asthma is a common clinical airway inflammatory disease triggered by antigens (1). It is mediated by $\mathrm{T}$ helper (Th) 2 cells and involves multiple inflammatory cells and molecules (2). Asthma is characterized by reversible airflow obstruction, high airway reactivity, increased secretion of airway mucus and immunoglobulin E, and eosinophil infiltration $(3,4)$. In recent decades, mortality due to asthma has increased in a number of countries, including the United States, Great Britain, Australia and New Zealand, making asthma a serious threat to public health globally (5).

Numerous studies have shown that oxidative stress plays an important role in the pathogenesis of asthma (6-8). When airway inflammation occurs, inflammatory mediators such as histamine and leukotriene are released. These mediators recruit and activate a variety of inflammatory cells, including eosinophils, neutrophils, lymphocytes, macrophages and platelets (9). The activation of inflammatory cells leads to the release of various free radicals and oxidative stress. Free radicals include reactive oxygen species (ROS) and reactive nitrogen species. These free radicals can cause serious damage or apoptosis of airway epithelial cells $(10,11)$. A number of studies have shown that oxidative damage by lipids, nucleic acids and proteins is widespread in the lungs of asthmatic mice sensitized by ovalbumin (OVA) (12-14). Oxidative stress indicators, such as nitrates and superoxide anions, are also increased in asthma patients (15). Multiple studies have detected elevated levels of hydrogen peroxide and nitric oxide in exhaled gases from asthma patients (16-18), which suggests potential oxidative injury.

As a free radical scavenger, Edaravone (Eda) scavenges free radicals and inhibits lipid peroxidation (19). It is currently used for the treatment of acute cerebral infarction (19). 
In recent years, numerous studies have found that it also has a protective effect against lung injury, due to its ability to eliminate ROS (20-22). Wang et al (22) found that Eda ameliorated LPS-induced pulmonary fibrosis by reducing lipopolysaccharide (LPS)-stimulated oxidative stress and activating transforming growth factor- $\beta 1 /$ mothers against decapentaplegic homolog 3 signaling. In another study, it was revealed that Eda attenuated oxidative stress and subsequent lung injury induced by liver reperfusion (23). Herbicide paraquat toxicity can cause severe oxidative injury in the lungs (24). In A549 cells treated with paraquat, administration of Eda decreased the levels of intracellular ROS and malondialdehyde (MDA) but increased the levels of superoxide dismutase (SOD) (25). In a canine lung transplantation model, Eda significantly decreased lung wet/dry ratios, MDA levels and myeloperoxidase activity (26). However, to the best of our knowledge the effects of Eda on asthma have not yet been investigated.

The nuclear factor erythroid 2-related factor 2 (Nrf2)/ antioxidant response element (ARE) signaling pathway is a defensive pathway in response to oxidative and chemical stress that is critical for the regulation of antioxidants and phase II detoxification enzymes. One example of an ARE is hemeoxygenase-1 (HO-1) (27). Under homeostatic conditions, Nrf2 binds to kelch-like ECH-associated protein-1 (Keap1) in the cytoplasm, remains inactive and is easily degraded. Under oxidative or chemical stress, Keap1 modification or Nrf2 phosphorylation results in activation of $\mathrm{Nrf} 2$ through its dissociation from Keap1. Activated Nrf 2 can then translocate into the nucleus and interact with HO-1 and other AREs (27). The expression of HO-1 is transactivated in response to stress. Several studies have shown that Eda may exert a protective effect through activation of the Nrf2/HO-1 pathway. Zhang et al (28) revealed that Eda reduced iron-mediated hydrocephalus and behavioral disorders in rats through Nrf2/HO-1 activation. In an animal model of cognitive damage induced by chronic cerebral hypoperfusion $(\mathrm{CCH})$, Eda reduced $\mathrm{CCH}$-induced cognitive damage and increased SOD activity and HO-1 levels, but decreased MDA levels in the hippocampus through activation of the Nrf2 pathway (29). Liu et al (30) found that Eda increased neuronal density and decreased neuronal damage induced by kainite, which was administrated in the right hippocampus CA3 region using the sereotactic technique and this enhanced the expression of Nrf2 and HO-1. However, all of these studies focused on the nervous system. Whether Eda can activate the Nrf2/HO-1 pathway in the lung remains to be elucidated.

In order to test the hypothesis that Eda may exert a protective effect against asthma, asthma was induced in a mouse model. The effects of Eda on airway responsiveness, cell and cytokine counts in bronchoalveolar lavage fluid (BALF), and levels of oxidative products and antioxidant enzymes in lung tissue were studied. The protein levels of Keap1/Nrf2 and HO-1 in lung tissue and their roles in the effects of Eda action were examined to investigate the underlying mechanisms.

\section{Materials and methods}

Animals. A total of 96 normal C57BL/6 male mice, 24 male Nrf2-deficient mice and 24 male HO-1-deficient mice with a C57BL/6/SV129 background (age, 6-7 weeks; weight, 20 \pm 2 g) were provided by the Experimental Animal Center of Jing'an District Centre Hospital of Fudan University and kept in a climate controlled room. The temperature of the animal room was kept at $24 \pm 3^{\circ} \mathrm{C}$ and the relative humidity was kept at 40-60\%. The study was approved by the Institutional Animal Care and Use Committee of the Jing'an District Centre Hospital of Fudan University (approval no. AS-17-3652).

After 1 week of adaptive feeding with access to food and water ad libitum, normal mice were randomly divided into six groups: Control group, OVA + saline group, OVA + Eda-low (L) group, OVA + Eda-moderate $(\mathrm{M})$ group, OVA + Eda-high (H) group, and Eda-H group, with 12 mice in each group. Mice in the control group received no treatment; mice in the OVA + saline group received OVA (cat. no. A5503; Grade V; Sigma-Aldrich; Merck KGaA) and intraperitoneal (i.p.) saline injection; mice in the OVA + Eda-L group received OVA and i.p. injection of a total of 14 low doses of Eda $(1 \mathrm{mg} / \mathrm{kg}$; cat. no. 443300; Sigma-Aldrich; Merck KGaA) in an equal volume of saline; mice in the OVA + Eda-M group received OVA and i.p. injection of a total of 14 moderate doses of Eda $(5 \mathrm{mg} / \mathrm{kg})$; mice in the OVA + Eda-H group received OVA and i.p. injection of a total of 14 high doses of Eda $(10 \mathrm{mg} / \mathrm{kg})$; mice in the Eda-H group received i.p. injection of a total of fourteen high doses of Eda $(10 \mathrm{mg} / \mathrm{kg})$ but were not treated with OVA. In a separate study, to explore the role of Nrf2 and HO-1 in response to Eda, normal and deficient mice were divided into control group (untreated group), OVA + Eda group, $\mathrm{OVA}+\mathrm{Eda}+\mathrm{Nrf}^{-/-}$group, $\mathrm{OVA}+\mathrm{Eda}+\mathrm{HO}-1^{-/}$group, $\mathrm{Nrf}^{-1-}$ group and $\mathrm{HO}-1^{-/-}$group, with 12 mice in each group. All Eda group mice received i.p. injection of a single moderate dose of Eda $(5 \mathrm{mg} / \mathrm{kg})$.

Asthma induction. On days 1, 8 and 15, mice in the OVA + saline group, OVA + Eda-L group, OVA + Eda-M group, OVA + Eda-H group, OVA + Eda + Nrf2 $2^{-/-}$group, and the OVA $+\mathrm{Eda}+\mathrm{HO}^{-1^{-/}}$group were injected i.p with $0.2 \mathrm{ml}$ of an antigen mixture (containing $50 \mu \mathrm{g}$ OVA, $150 \mu 110 \%$ aluminum hydroxide (cat. no. 1.01091; Sigma-Aldrich; Merck $\mathrm{KGaA}$ ) and $50 \mu \mathrm{l}$ physiological saline). Beginning on day 22, mice inhaled 1\% OVA for 30 min daily in a plastic box using a nebulizer (emka TECHNOLOGIES) for 14 days as an asthma challenge. I.p Eda was administered $30 \mathrm{~min}$ before each challenge for 14 days. The study period was 36 days.

BALF collection and cell count. Twenty-four $\mathrm{h}$ after the last OVA challenge, mice were anesthetized with sodium pentobarbital (100 mg/kg, i.p.) and pancuronium bromide $(6 \mu \mathrm{g} / \mathrm{g}$, i.p.), and the lungs were exposed by thoracotomy. After these procedures were completed, the mice were sacrificed by cervical dislocation. The spinal column was quickly dislocated from the brain by pressing the base of the skull and pulling backward on the tail to provide a fast and painless death for the mice. BALF was then collected from the left lung. BALF was centrifuged at $110 \mathrm{xg}$ for $10 \mathrm{~min}$ at $4^{\circ} \mathrm{C}$, and the cells were resuspended in $1 \mathrm{ml} \mathrm{Hank's} \mathrm{solution} \mathrm{(cat.} \mathrm{no.} \mathrm{C0218;} \mathrm{Beyotime}$ Institute of Biotechnology). An aliquot of the cell suspension $(100 \mu \mathrm{l})$ was put into a blood cell counter to determine the total number of cells and a further $200 \mu \mathrm{l}$ taken for Diff-Quick staining (Baxter Diagnostics, Inc.) (31). At least 200 cells were counted in each sample and classified as eosinophils, 
lymphocytes and neutrophils according to their morphological characteristics (32).

Measurement of airway responsiveness to inhaled methacholine (Mch). The procedure for the measurement of airway responsiveness to inhaled Mch has been described in a previous publication (33). Briefly, mice were anesthetized with sodium pentobarbital (100 $\mathrm{mg} / \mathrm{kg}$, i.p.) and pancuronium bromide $(6 \mu \mathrm{g} / \mathrm{g}$, i.p.) and then intubated via tracheostomy and mechanically ventilated in a plethysmograph chamber. Intraesophageal and airway pressures were measured with a dedicated pressure transducer (Validyne DP45; Buxco ${ }^{\circledR}$, $\mathrm{DSI}^{\mathrm{TM}}$, Harvard Bioscience, Inc.). The signals generated were preamplified (MaxII, Buxco ${ }^{\circledR}$, DSI $^{\mathrm{TM}}$, Harvard Bioscience, Inc.) and captured with computer software (Biosystem XA; version 2.7.9; Buxco ${ }^{\circledR}$; DSI ${ }^{\mathrm{TM}}$; Harvard Bioscience, Inc.). The baseline readings were taken and averaged for $3 \mathrm{~min}$. Respiratory resistance (RR) was measured in response to increasing doses of inhaled Mch (1, 2 and $4 \mathrm{mg} / \mathrm{ml})$, and $2 \mu \mathrm{l}$ of each dose of methacholine was delivered through an ultrasonic nebulizer over 10 consecutive breaths. Tidal volume was increased to $350 \mu \mathrm{l}$, and respiratory rate was reduced to 100 breaths $/ \mathrm{min}$ during each nebulization and then returned to pre-nebulization values. RR to airflow was determined continuously for $10 \mathrm{~min}$, and the peak responses following each dose of Mch were obtained. After these procedures were completed, the mice were sacrificed by cervical dislocation, as described above.

BALF cytokine measurement. BALF was centrifuged at $110 \mathrm{x} \mathrm{g}$ for $10 \mathrm{~min}$ at $4^{\circ} \mathrm{C}$. The levels of inflammatory cytokines interleukin (IL)-6 (cat. no. PI326; Beyotime Institute of Biotechnology), IL-13 (cat. no. ab219634, Abcam), interferon (IFN) $-\gamma$ (cat. no. PI508; Beyotime Institute of Biotechnology) and tumor necrosis factor (TNF)- $\alpha$ (cat. no. PT512; Beyotime Institute of Biotechnology) were measured in the BALF supernatants using commercial ELISA kits according to the manufacturer's instructions. Briefly, the monoclonal capture antibody was pre-coated on the ELISA plate. When the sample was added, the target protein therein bound to the capture antibody. A biotinylated anti-target protein antibody was added, which bound to the target protein to form a sandwich immune complex. Subsequently, horseradish peroxidase (HRP)-labeled streptavidin was added, which bound to the sandwich immune complex. Finally, the developer 3,3',5,5'-tetramethylbenzidine (TMB) solution was added. HRP then catalyzed the oxidation of the colorless chromogenic agent TMB to a blue substance, which became yellow after the addition of the stop solution. The absorbance at $450 \mathrm{~nm}$ was then measured using a Multi-Detection Microplate Reader (BioTek Instruments, Inc.). A standard curve was generated using known concentrations of each substance and used to compare with the absorbance values of the sample, allowing the concentration of cytokines in the sample to be interpolated.

Histopathology evaluation. At $24 \mathrm{~h}$ after the last OVA challenge, the left lobes of lung tissue samples were collected and fixed with $10 \%$ formalin for $48 \mathrm{~h}$ at room temperature. Lung tissues were cut into $5 \mu \mathrm{m}$-thick sections and mounted onto slides, which were stained with hematoxylin and eosin for $5 \mathrm{~min}$ at room temperature. These slides were then randomly and blindly examined by a pathologist to evaluate the histopathological appearance (three slides and four fields of view per sample; magnification, x200).

Measurement of ROS, total antioxidant capacity (T-AOC) and $M D A$ in the lung. Levels of intracellular ROS were measured through oxidative conversion of 2',7'-dichlorofluorescein diacetate (DCFH-DA) to the fluorescent compound dichlorofluorescin (34). In brief, $24 \mathrm{~h}$ after the last OVA challenge, lung tissue samples were taken, weighed (50 mg) and homogenized on ice with ice-cold RIPA lysis buffer (cat. no. P0013B; Beyotime Institute of Biotechnology) for $30 \mathrm{~min}$. After centrifugation $\left(16,000 \mathrm{x} \mathrm{g}, 10 \mathrm{~min}\right.$ at $\left.4^{\circ} \mathrm{C}\right)$, lung homogenates were incubated with PBS containing $15 \mu \mathrm{M}$ DCFH-DA (Nanjing Jiancheng Bioengineering Institute) for $30 \mathrm{~min}$ at $37^{\circ} \mathrm{C}$ to label intracellular ROS. The cellular fluorescence was determined using a microplate reader (Promega Corporation) at 490 and $520 \mathrm{~nm}$. The total antioxidant capacity (T-AOC) was determined using a detection kit (cat. no. A015-2-1; Nanjing Jiancheng Bioengineering Institute) and measuring the absorbance at $520 \mathrm{~nm}$. The MDA content was measured using a thiobarbituric acid reactive substances assay in accordance with the manufacturer's instructions (Nanjing Jiancheng Bioengineering Institute), and the absorbance was measured at a wavelength of $532 \mathrm{~nm}$ (35).

Measurement of SOD, catalase (CAT) and glutathione peroxidase (GSH-Px) in the lung. A period of $24 \mathrm{~h}$ after the last OVA challenge, lung tissue samples were taken, weighed (50 mg) and homogenized on ice with RIPA ice-cold lysis buffer (cat. no. P0013B; Beyotime Institute of Biotechnology) for $30 \mathrm{~min}$. After centrifugation $\left(16,000 \mathrm{x} \mathrm{g}, 10 \mathrm{~min}\right.$ at $\left.4^{\circ} \mathrm{C}\right)$, the activities of SOD, CAT and GSH-Px in lung homogenate were measured using commercial kits (Beyotime Institute of Biotechnology), on 96-well plates, according to the manufacturer's instructions. SOD activity was detected with a total SOD assay kit with 2-(2-methoxy-4-nitrophenyl)-3-(4-nitro phenyl)-5-(2,4-disulfophenyl)-2H-tetrazolium, monosodium salt (cat. no. S0101). The absorbance at $450 \mathrm{~nm}$ was then measured using an HT Multi-Detection Microplate Reader (BioTek Instruments, Inc.). CAT activity was measured with a catalase assay kit (cat. no. S0051). The plate was measured at $520 \mathrm{~nm}$. GPx activity was measured with a GPx assay kit (cat. no. S0056). The plate was measured at $340 \mathrm{~nm}$. Finally, the activities of SOD, CAT and GSH-Px were calculated by plotting standard curves and comparing the absorbance values of the samples and the standards.

Western blot analysis. Twenty-four h after the last OVA challenge, lung tissue samples were taken, weighed (50 mg) and homogenized on ice with ice-cold lysis buffer (cat. no. P0013; Beyotime Institute of Biotechnology) for $30 \mathrm{~min}$. After centrifugation $\left(16,000 \mathrm{x} \mathrm{g}, 10 \mathrm{~min}\right.$ at $\left.4^{\circ} \mathrm{C}\right)$, the supernatant was collected. Proteins in the supernatant were measured using the BCA method (cat. no. P0010; Beyotime Institute of Biotechnology). Proteins were separated using $10 \%$ SDS-PAGE and then transferred to PVDF membranes (EMD Millipore). The blotted membranes were blocked with $5 \%$ non-fat dry milk (Beyotime Institute of Biotechnology). A period of $2 \mathrm{~h}$ later (at room temperature), the membranes 
Table I. Airway responsiveness to Mch.

\begin{tabular}{lcccc}
\hline & \multicolumn{4}{c}{ RR, \% of baseline } \\
\cline { 2 - 5 } Group & $\mathrm{NS}$ & Mch $(1 \mathrm{mg} / \mathrm{ml})$ & Mch $(2 \mathrm{mg} / \mathrm{ml})$ & $\mathrm{Mch}(4 \mathrm{mg} / \mathrm{ml})$ \\
\hline Control & $102.6 \pm 10.5$ & $113.6 \pm 15.8$ & $122.7 \pm 10.4$ & $152.4 \pm 13.2$ \\
OVA + saline & $111.2 \pm 19.5$ & $143.7 \pm 15.1^{\mathrm{a}}$ & $188.5 \pm 12.6^{\mathrm{a}}$ & $223.1 \pm 15.2^{\mathrm{a}}$ \\
OVA + Eda-L & $105.6 \pm 16.2$ & $129.5 \pm 14.7$ & $172.9 \pm 16.2$ & $221.4 \pm 12.9$ \\
OVA + Eda-M & $103.7 \pm 15.5$ & $112.2 \pm 11.4^{\mathrm{b}}$ & $123.4 \pm 11.2^{\mathrm{b}}$ & $120.3 \pm 12.8^{\mathrm{b}}$ \\
OVA + Eda-H & $103.3 \pm 15.7$ & $108.5 \pm 10.4^{\mathrm{b}}$ & $123.2 \pm 11.9$ & $144.6 \pm 20.8^{\mathrm{b}}$ \\
Eda-H & $106.8 \pm 16.1$ & $114.1 \pm 12.2$ & $159.7 \pm 18.8$ \\
\hline
\end{tabular}

Data are presented as the mean $\pm \mathrm{SD}$ (the mean $\%$ of baseline). ${ }^{\mathrm{a}} \mathrm{P}<0.05$ compared with control; ${ }^{\mathrm{b}} \mathrm{P}<0.05$ compared with $\mathrm{OVA}+$ saline. Mch, methacholine; NS, normal saline vehicle; OVA, ovalbumin; RR, respiratory resistance; Eda, edaravone; L, low dose; M, moderate dose; $\mathrm{H}$, high dose.

were washed with $0.2 \%$ Tween-20 in TBS (TBST) and incubated with anti-Keap1 (1:1,000; cat. no. 4678), anti-Nrf2 (1:500; cat. no. 4399), anti-HO-1 (1:1,000; cat. no. 70081) and anti- $\beta$-actin $(1: 1,000$; cat. no. 3700$)$ antibodies at $4^{\circ} \mathrm{C}$ overnight. The membranes were then washed with TBST and probed with HRP-conjugated anti-rabbit IgG (1:5,000; cat. no. 7074) for $1 \mathrm{~h}$ at room temperature. All antibodies were supplied by CST Biological Reagents Co., Ltd. After antibody incubations the blots were washed with TBST and visualized with a peroxidase/luminol enhanced chemiluminescence reagent (Thermo Fisher Scientific, Inc.) using Quantity One software (version 4.6.2; Bio-Rad Laboratories, Inc.).

Statistical analysis. Data are presented as the mean \pm SEM (three repeats). Statistical tests performed were one-way analysis of variance with Tukey-Kramer or Tamhane's tests by SPSS 17.0 (SPSS Inc.). GraphPad Prism 5 (GraphPad Software, Inc.) software was used for plotting graphs. $\mathrm{P}<0.05$ was considered to indicate a statistically significant difference.

\section{Results}

Eda reduces airway responsiveness to Mch. To measure the effect of Eda on asthma, mice were treated with different doses of Eda and the airway responsiveness of the mice to Mch was measured. As shown in Table I, in the control group, the airway responsiveness to Mch gradually increased as the concentration of Mch increased. In the animal model of asthma, the airway responsiveness to Mch was significantly higher than in the control group. In the OVA + Eda-L group, the airway responsiveness to Mch was not significantly different when compared with OVA + saline. In the OVA + Eda-M and OVA + Eda-H groups, however, the airway responsiveness to Mch was significantly reduced when compared with OVA + saline. Treatment with Eda-H alone did not affect airway responsiveness compared with the control.

Eda reduces cell count in BALF. Cell counts, including total cells, eosinophils, lymphocytes and neutrophils, were subsequently measured in BALF. As shown in Table II, all cell counts were significantly increased by OVA treatment when compared with control. In the OVA + Eda-M and OVA + Eda-H groups, total cells, eosinophils, lymphocytes and neutrophils in BALF were significantly reduced when compared with the OVA + saline group. Treatment with Eda-H alone did not affect the BALF cell count compared with the control.

Eda reduces the levels of cytokines in BALF. To measure the effect of Eda on lung inflammation, the levels of IL-6, IL-13, IFN- $\gamma$ and TNF- $\alpha$ were examined in BALF. As shown in Table III, the levels of IL-6, IL-13, IFN- $\gamma$ and TNF- $\alpha$ in BALF were significantly increased by OVA treatment compared with the control. In the OVA + Eda-M and OVA + Eda-H groups, however, these parameters were all significantly reduced when compared with the OVA + saline group. Treatment with Eda-H alone did not significantly affect cytokine levels compared with the control.

Eda attenuates lung histopathological changes caused by $O V A$. Fig. 1 shows histopathological changes in the mice. The normal control group showed normal lung architecture. There was no perivascular edema or peribronchial inflammation. Slides from the OVA + saline and OVA + Eda-L groups showed perivascular edema and peribronchial infiltration. The alveolar spaces were filled with alveolar macrophages and inflammatory cells. In the OVA + Eda-M and OVA + Eda-H groups, however, there was a marked decrease in perivascular edema, peribronchial inflammation and macrophage infiltration in the alveolar space compared to the OVA + saline group. Lung histopathology was not significantly changed in the Eda-H group when compared with the control group.

Eda attenuates oxidative stress in the lung. To measure oxidative stress in the lung, the levels of ROS, T-AOC and MDA were examined in lung tissue. As shown in Fig. 2A, ROS level was significantly increased in the OVA + saline group compared with the control group. In the OVA + Eda-M and OVA + Eda-H groups, ROS levels were significantly decreased compared with the OVA + saline group. Treatment with Eda-H alone did not significantly affect ROS content. By contrast, T-AOC was significantly decreased in the OVA + saline group compared with the control group but greatly increased in 
Table II. Cell count in BALF.

\begin{tabular}{lcccc}
\hline & \multicolumn{3}{c}{ Cell count $\left(1 \times 10^{4} / \mathrm{ml}\right)$} \\
\cline { 2 - 5 } Group & Total cells & Eosinophils & Lymphocytes & Neutrophils \\
\hline Control & $7.51 \pm 1.61$ & $0.17 \pm 0.05$ & $0.44 \pm 0.06$ & $0.35 \pm 0.05$ \\
OVA + saline & $21.82 \pm 1.92^{\mathrm{a}}$ & $3.52 \pm 0.52^{\mathrm{a}}$ & $6.89 \pm 0.84^{\mathrm{a}}$ & $4^{\mathrm{a}}$ \\
OVA + Eda-L & $20.65 \pm 1.54$ & $3.44 \pm 0.62$ & $6.54 \pm 0.78$ & $4.29 \pm 0.63$ \\
OVA +Eda-M & $14.54 \pm 1.35^{\mathrm{b}}$ & $2.41 \pm 0.52^{\mathrm{b}}$ & $3.56 \pm 0.48^{\mathrm{b}}$ & $2.31 \pm 0.41^{\mathrm{b}}$ \\
OVA + Eda-H & $10.62 \pm 1.56^{\mathrm{b}}$ & $2.03 \pm 0.61^{\mathrm{b}}$ & $3.25 \pm 0.49^{\mathrm{b}}$ & \\
Eda-H & $7.44 \pm 1.53$ & $0.16 \pm 0.08$ & $0.49 \pm 0.08$ & $0.39 \pm 0.08$ \\
\hline
\end{tabular}

Data are presented as the mean $\pm \mathrm{SD} .{ }^{a} \mathrm{P}<0.05$ compared to control; ${ }^{b} \mathrm{P}<0.05$ compared to OVA + saline. OVA, ovalbumin; Eda, edaravone; $\mathrm{L}$, low dose; $\mathrm{M}$, moderate dose; $\mathrm{H}$, high dose.

Table III. Levels of cytokines in BALF.

\begin{tabular}{|c|c|c|c|c|}
\hline \multirow[b]{2}{*}{ Group } & \multicolumn{4}{|c|}{ Levels of cytokines (ng/l) } \\
\hline & IL-6 & IL-13 & IFN- $\gamma$ & $\mathrm{TNF}-\alpha$ \\
\hline Control & $15.8 \pm 2.2$ & $18.6 \pm 2.7$ & $26.2 \pm 6.3$ & $11.3 \pm 3.3$ \\
\hline OVA + saline & $29.6 \pm 3.1^{\mathrm{a}}$ & $36.2 \pm 3.4^{\mathrm{a}}$ & $56.3 \pm 10.2^{\mathrm{a}}$ & $29.6 \pm 3.5^{\mathrm{a}}$ \\
\hline OVA + Eda-L & $28.5 \pm 3.9$ & $35.1 \pm 3.3$ & $52.3 \pm 8.5$ & $28.4 \pm 2.8$ \\
\hline OVA + Eda-M & $20.3 \pm 3.5^{\mathrm{b}}$ & $23.6 \pm 3.1^{\mathrm{b}}$ & $32.6 \pm 6.9^{b}$ & $20.6 \pm 3.7^{b}$ \\
\hline $\mathrm{OVA}+\mathrm{Eda}-\mathrm{H}$ & $16.5 \pm 2.8^{b}$ & $20.2 \pm 2.5^{\mathrm{b}}$ & $28.5 \pm 5.5^{\mathrm{b}}$ & $16.9 \pm 3.2^{\mathrm{b}}$ \\
\hline Eda-H & $15.4 \pm 2.4$ & $19.5 \pm 2.1$ & $23.6 \pm 6.7$ & $13.4 \pm 3.1$ \\
\hline
\end{tabular}

Data are presented as the mean $\pm \mathrm{SD} .{ }^{\mathrm{a}} \mathrm{P}<0.05$ compared with control; ${ }^{\mathrm{b}} \mathrm{P}<0.05$ compared with $\mathrm{OVA}+$ saline. IFN- $\gamma$, interferon- $\gamma$; IL, interleukin; OVA, ovalbumin; TNF- $\alpha$, tumor necrosis factor- $\alpha$; Eda, edaravone; L, low dose; M, moderate dose; H, high dose.

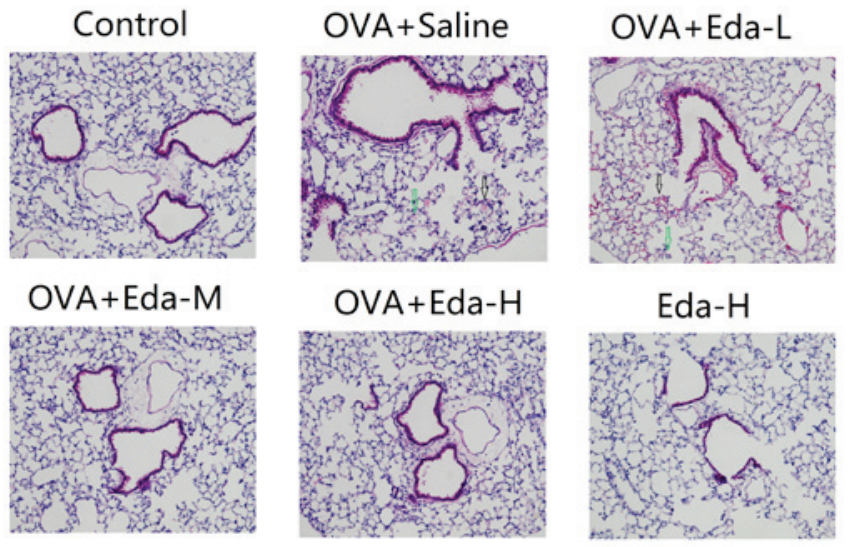

Figure 1. Effects of Eda on lung histopathology. Lung slides were stained with hematoxylin and eosin (magnification, x200). Black arrow, lung edema; green arrow, alveolar macrophages and inflammatory cells; Eda, edaravone; OVA, ovalbumin; L, low dose; M, moderate dose; H, high dose.

$\mathrm{OVA}+\mathrm{Eda}-\mathrm{M}$ and OVA + Eda-H groups compared with the OVA + saline group (Fig. 2B). Treatment with Eda-H alone significantly increased T-AOC level compared to the control. MDA levels in lung tissues were significantly enhanced by OVA treatment but greatly decreased by treatment with moderate and high dosages of Eda when compared with the OVA + Saline group (Fig. 2C). Treatment with Eda-H alone did not significantly affect MDA content compared with the control group.

Eda increases the levels of antioxidative enzymes in the lung. To measure the effect of Eda on antioxidative enzymes in the lung, the levels of antioxidative enzymes (SOD, CAT, GSH-Px) were examined in lung tissues. The results showed that SOD was greatly decreased in the OVA + saline group but recovered with treatment with $\mathrm{M}$ and $\mathrm{H}$ doses of Eda (Fig. 3A). The levels of CAT (Fig. 3B) and GSH-Px (Fig. 3C) were also significantly decreased in the OVA + saline group when compared with the control but addition of Eda had no additional effect.

Eda activates the Keapl/Nrf2 pathway and HO-1 expression in the lung. To explore the effect of Eda on the Keap1/Nrf2 pathway and HO-1 expression in the lung, the expression of Keap1, Nrf2 and HO-1 was measured in lung tissue by western blotting, as shown in Fig. 4. The results showed that after mice were treated with moderate and high doses of Eda, Keap1/Nrf2 ratio was significantly reduced, and Nrf2 (normalized to $\beta$-actin) and HO-1 (normalized to $\beta$-actin) expression was significantly increased, indicating that the Keap1/Nrf2 pathway was activated. 

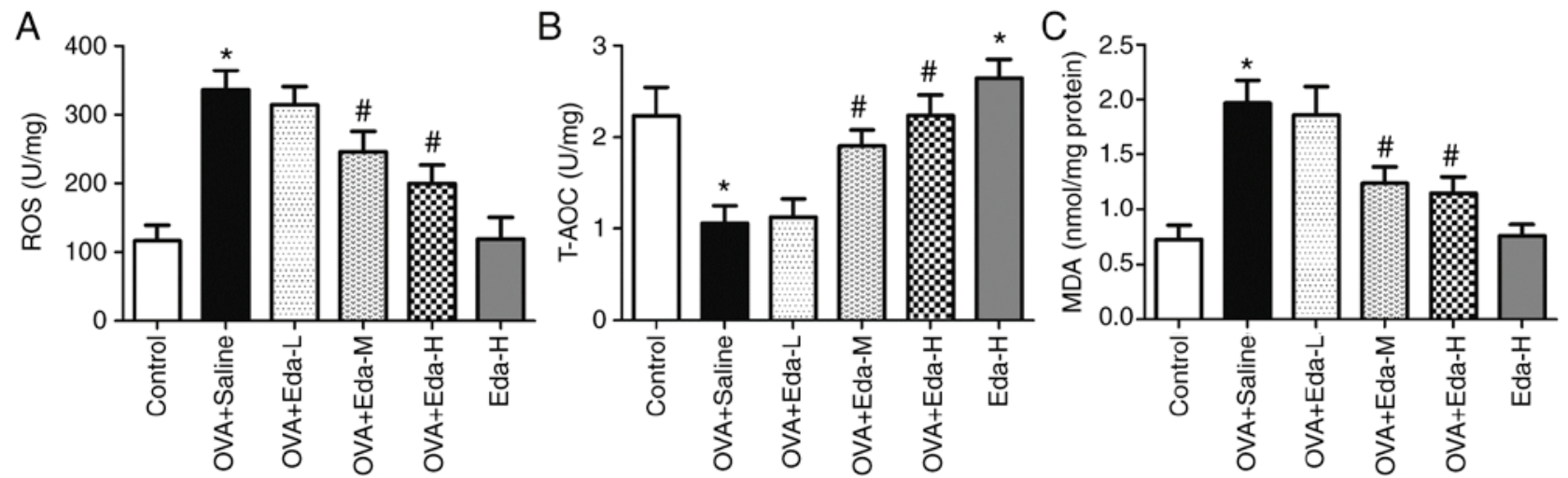

Figure 2. Effects of Eda on oxidative stress in the lung. To measure the effects of Eda on oxidative stress in lungs, the levels of (A) ROS, (B) T-AOC and (C) MDA in lung tissues were examined. Data are presented as the mean \pm SEM. "P $<0.05$ compared with control; " $\mathrm{P}<0.05$ compared with OVA + saline. MDA, malondialdehyde; OVA, ovalbumin; ROS, reactive oxygen species; T-AOC, total antioxidant capacity; Eda, edaravone; L, low dose; M, moderate dose; $\mathrm{H}$, high dose.
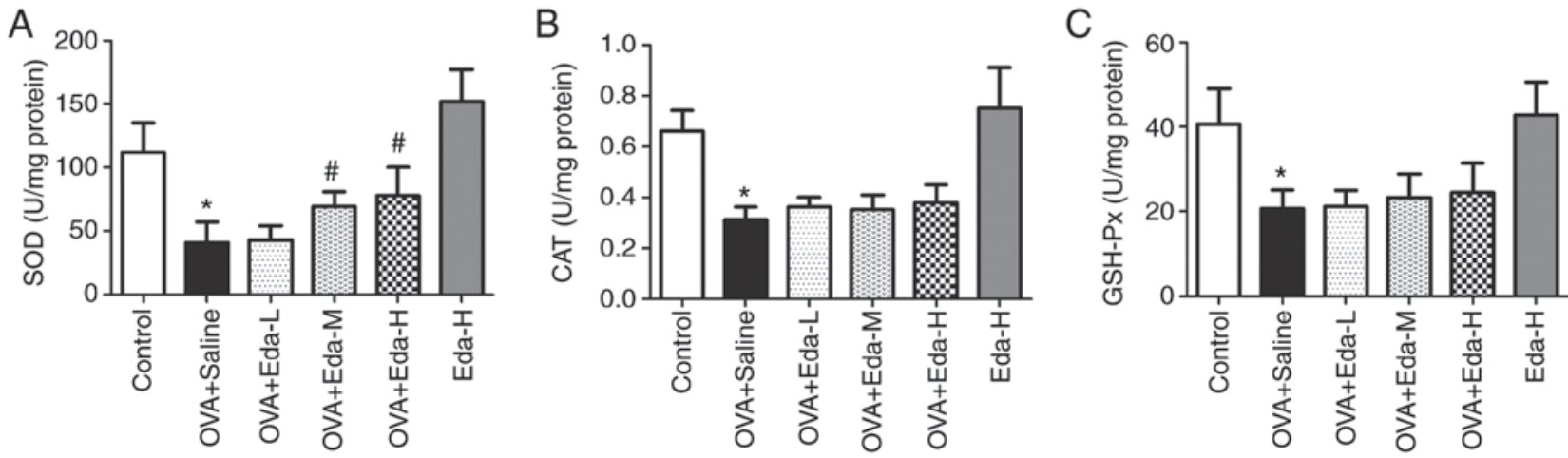

Figure 3. Effects of Eda on antioxidative enzymes in the lung. To measure the effects of Eda on antioxidative enzymes in lungs, the levels of (A) SOD, (B) CAT and (C) GSH-Px in lung tissues were examined. Data are presented as the mean \pm SEM. ${ }^{*} \mathrm{P}<0.05$ compared with control; ${ }^{*} \mathrm{P}<0.05$ compared with OVA + saline. CAT, catalase; GSH-Px, glutathione peroxidase; OVA, ovalbumin; SOD, superoxide dismutase; Eda, edaravone; L, low dose; M, moderate dose; H, high dose.
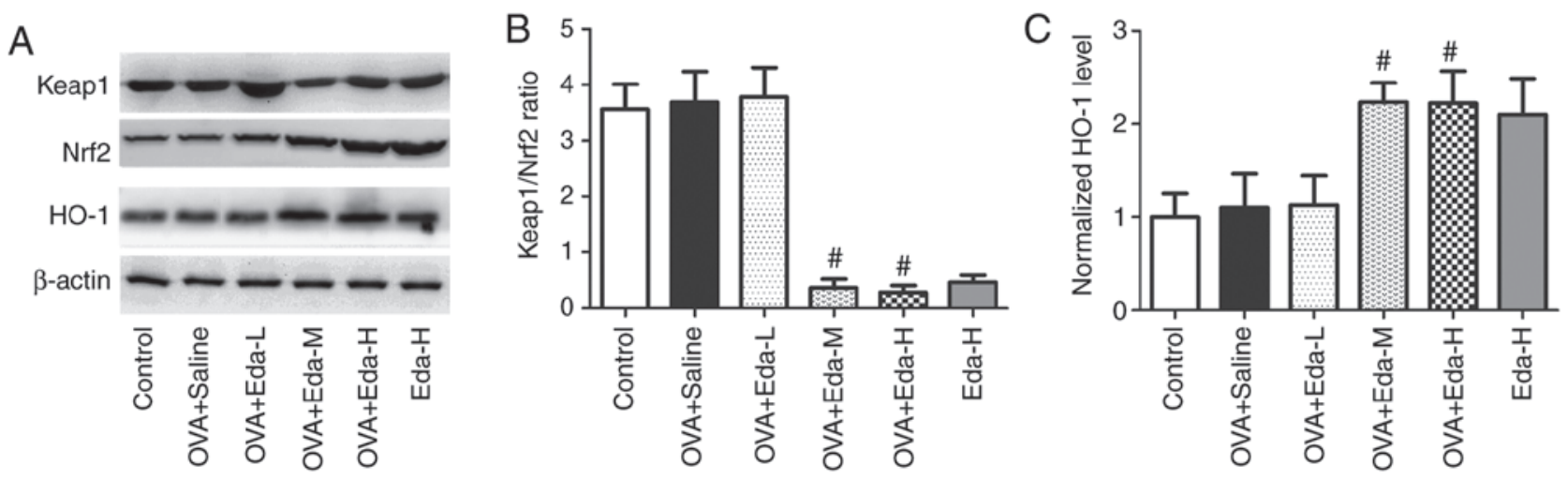

Figure 4. Effects of Eda on the expression of Keap1, Nrf2 and HO-1 in lungs. To explore the effect of Eda on the Keap1/Nrf2 pathway and HO-1 expression in lungs, the expression of Keap1, Nrf2 and HO-1 was measured in lungs by western blotting. (A) representative bands of Keap1, Nrf2 and HO-1. (B) Results of Keap1/Nrf2 ratio. (C) Results of normalized HO-1 levels to $\beta$-actin. Data are presented as the mean \pm SEM. $\mathrm{P}<0.05$ compared with OVA + saline. HO-1, hemeoxygenase-1; Keap1, kelch-like ECH-associated protein-1; OVA, ovalbumin; Nrf2, nuclear factor erythroid 2-related factor 2; Eda, edaravone; L, low dose; $\mathrm{M}$, moderate dose; $\mathrm{H}$, high dose.

Nrf2 or HO-1 deficiency abolishes the effect of Eda on airway responsiveness to Mch. To explore the role of the Keap1/Nrf2 pathway and HO-1 in the effect of Eda on airway responsiveness to Mch, normal, $\mathrm{Nrf2}^{-/}$and $\mathrm{HO}-1^{-/}$mice were treated with Eda and their airway responsiveness to Mch was measured.
As shown in Table IV, the airway responsiveness of Nrf2-1and $\mathrm{HO}-1^{-/}$mice to Mch was significantly higher than that of normal mice treated with OVA and Eda $(\mathrm{P}<0.05)$. The airway responsiveness of $\mathrm{Nrf}^{-/}$and $\mathrm{HO}-1^{-/}$mice treated with Eda was increased to a similar level as the OVA + saline group. 
Table IV. Airway responsiveness to Mch in $\mathrm{Nrf} 2^{-/-}$or $\mathrm{HO}-1^{-/-}$mice.

\begin{tabular}{|c|c|c|c|c|}
\hline \multirow[b]{2}{*}{ Group } & \multicolumn{4}{|c|}{$\mathrm{RR}, \%$ of baseline } \\
\hline & NS & $\operatorname{Mch}(1 \mathrm{mg} / \mathrm{ml})$ & $\operatorname{Mch}(2 \mathrm{mg} / \mathrm{ml})$ & $\operatorname{Mch}(4 \mathrm{mg} / \mathrm{ml})$ \\
\hline Control & $103.6 \pm 11.5$ & $119.3 \pm 12.8$ & $124.5 \pm 16.7$ & $158.9 \pm 18.2$ \\
\hline OVA + saline & $110.1 \pm 15.2$ & $135.1 \pm 14.2$ & $174.3 \pm 10.9$ & $219.3 \pm 14.5$ \\
\hline $\mathrm{OVA}+\mathrm{Eda}$ & $104.5 \pm 12.3$ & $117.2 \pm 10.6$ & $128.6 \pm 12.4$ & $156.9 \pm 17.1$ \\
\hline $\mathrm{OVA}+\mathrm{Eda}+\mathrm{Nrf}^{-/-}$ & $113.6 \pm 15.7$ & $136.9 \pm 13.5^{\mathrm{a}}$ & $176.75 \pm 12.4^{\mathrm{a}}$ & $216.8 \pm 14.1^{\mathrm{a}}$ \\
\hline $\mathrm{OVA}+\mathrm{Eda}+\mathrm{HO}-1^{-/-}$ & $115.2 \pm 16.5$ & $136.4 \pm 13.2^{\mathrm{a}}$ & $171.4 \pm 10.7^{\mathrm{a}}$ & $214.2 \pm 13.3^{\mathrm{a}}$ \\
\hline $\mathrm{Nrf} 2^{-/-}$ & $103.7 \pm 12.4$ & $111.5 \pm 11.5$ & $120.4 \pm 10.7$ & $153.4 \pm 15.2$ \\
\hline $\mathrm{HO}-1^{-/-}$ & $106.4 \pm 11.4$ & $115.2 \pm 13.2$ & $121.1 \pm 12.1$ & $141.1 \pm 12.1$ \\
\hline
\end{tabular}

Data are presented as the mean $\pm \mathrm{SD}$ (the mean $\%$ of baseline). ${ }^{\mathrm{a}} \mathrm{P}<0.05$ compared to OVA + Eda. HO-1, hemeoxygenase- 1 ; Nrf2, nuclear factor erythroid 2-related factor 2; Mch, methacholine; NS, normal saline vehicle; OVA, ovalbumin; RR, respiratory resistance; Eda, edaravone; $\mathrm{L}$, low dose; $\mathrm{M}$, moderate dose; $\mathrm{H}$, high dose.

The airway responsiveness of $\mathrm{Nrf}^{-/-}$mice and $\mathrm{HO}-1^{-/-}$mice without OVA treatment was not significantly different from control mice.

\section{Discussion}

In recent years, the hypotheses of free radical cell injury and oxidant/antioxidant imbalance in asthma have attracted widespread attention (12-14). It was indicated that the increased ROS activated $\mathrm{NF}-\kappa \mathrm{B}$ activity, enhanced asthmatic inflammatory response and induced glucocorticoid resistance (36). The basic features of bronchial asthma include chronic airway inflammation, airway hyper-responsiveness, and airway wall structural changes induced by long-term inflammatory conditions (37). Frossi et al (38) found that oxidative stress upregulated Th2-mediated inflammatory responses, increased the severity of asthma, enhanced bronchial hyperresponsiveness and promoted airway remodeling. Therefore, the use of antioxidants to correct an imbalance in the oxidant/antioxidant system in asthma patients may protect the lung from oxidative damage and mitigate inflammatory reactions and airway remodeling. The present study established an animal model of asthma with OVA and examined the effect of Eda against it. It was shown that Eda reduced airway responsiveness to Mch when compared with normal saline. The numbers of total cells, eosinophils, lymphocytes and neutrophils in BALF were also significantly reduced by Eda compared with saline, as were the levels of cytokines (IL-6, IL-13, IFN- $\gamma$ and TNF- $\alpha$ ) in BALF. Histopathology showed that Eda alleviated perivascular edema, peribronchial inflammation and macrophage infiltration in the alveolar space caused by OVA compared with saline. These results revealed that Eda effectively reduced the degree of asthma and inflammatory reaction in lungs. Other studies have shown the anti-inflammatory effects of Eda in the lung. In LPS-induced lung injury, Eda markedly reduced polymorphonuclear leukocyte infiltration and TNF- $\alpha$ and IL-6 concentrations in both serum and BALF $(20,39)$. It was also shown to suppress LPS-induced NF- $\kappa \mathrm{B}$ activation and cyclo-oxygenase-2 expression (20). In an animal model of lung injury induced by black gunpowder smog, Eda significantly reduced myeloperoxidase activity and alleviated neutrophil infiltration of the lung (40). In the lung tissues of rats with myocardial ischemia reperfusion injury, Eda significantly decreased the concentration of serum creatine kinase isoenzyme, the lung permeability index, $\beta$-defensin- 2 mRNA expression and TNF- $\alpha$ protein expression (41). Taken together, these results suggest that the protective effect of Eda against lung asthma may be related to a suppression of the inflammatory response.

Oxidative stress has been shown to be closely related to the development of asthma and is involved in airway inflammation, airway hyper-responsiveness, increased airway vascular permeability, tissue damage and airway remodeling (39-44). Multiple ROS have strong oxidative activity and may cause tissue damage, leading to smooth muscle contraction and airway hyper-responsiveness (45). Oxidative stress can preferentially induce $\mathrm{T}$ cell activation to $\mathrm{Th} 2$, resulting in Th1/Th2 unbalance (46). Frossi et al (38) found that oxidative stress aggravated the severity of asthma, increased bronchial hyper-responsiveness, and promoted airway remodeling. In mice where oxidative stress-related genes were knocked out, an asthma and airway remodeling model could not be established (47). After antioxidant enzymes were administered for 28 days, the lung function of asthma patients was significantly improved (48). Studies have confirmed that alveolar epithelial cells in asthma patients are more susceptible to oxidative stress, apoptosis and increased permeability (49). A growing body of evidence supports the theory that oxidative stress mediated by exogenous and endogenous ROS is an important factor in the initiation of inflammatory mediators and cell damage (50). To measure oxidative stress in the lung, the present study examined the levels of ROS, T-AOC and MDA and antioxidative enzymes in lung tissue. The analyses revealed that Eda significantly decreased the levels of ROS, T-AOC and MDA compared with controls, and restored the levels of the antioxidative enzyme, SOD. A study has shown that levels of peroxide, MDA and conjugated diene were increased in the bronchial secretions of patients with asthma, while catalase activity was significantly reduced (51). As a ROS metabolite, MDA reflects lipid peroxidation, protein denaturation and impaired integrity of endothelial cells (52-54). Under asthma 
attacks, more peroxide, MDA, conjugated diene and other substances were found, and SOD activity decreased significantly $(13,55)$. SOD is an important enzyme in the endogenous antioxidative system in lung tissue (56). SOD levels in lung tissues indirectly reflect the degree of damage to lung cells by ROS (56). The results of oxidative stress and antioxidative enzymes in the present study indicated that Eda may alleviate the severity of asthma by suppressing oxidative injury and restoring the antioxidative enzyme, SOD.

It has been shown that Eda may exert a protective effect through the Nrf2/HO-1 pathway (28-30). Li et al (57) revealed that Eda protects against hyperosmolarity-induced oxidative stress and apoptosis in primary human corneal epithelial cells by increasing the expression of Nrf2 and its target genes, such as HO-1 and GPx-1. Eda was also found to upregulate the gene expression of $\mathrm{Nrf} 2 / \mathrm{HO}-1$ and alleviate cisplatin-induced neurobehavioral deficits (58). To explore the effect of Eda on the Keap1/Nrf2 pathway and HO-1 expression in lungs, the present study measured the expression levels of Keap1, Nrf2 and HO-1 by western blotting. The results showed that Eda significantly decreased the Keap1/Nrf2 ratio, when compared with controls, indicating that the Keap1/Nrf2 pathway was activated. Eda also greatly increased $\mathrm{HO}-1$ expression, suggesting that HO-1 may also participate in the anti-asthmatic action of Eda. It was hypothesized that the levels of Nrf2 and HO-1 may be decreased in response to OVA, but the results showed that they were not significantly changed by it. The reason for this may be that the levels of Nrf2 and HO-1, which is an ARE, can be activated in response to stress and inflammatory insults. Under oxidative or chemical stress, activated Nrf2 translocates into the nucleus and interacts with AREs (59). The expression of cytoprotective target genes, including phase II detoxifying enzymes, antioxidant proteins and molecular proteasomes/chaperones, is then transactivated in response to the stress (60). As a result, in the present study, there may be a balance between the consumption and production of Nrf2 and HO-1 during OVA insult, making it appear that the levels of Nrf2 and HO-1 were unchanged. Treatment with Eda, however, may further activate the Nrf2/ARE pathway, increasing the levels of Nrf2 and HO-1. To confirm the role of the Keap1/Nrf2 pathway and HO-1 on the effect of Eda, normal, $\mathrm{Nrf}^{-/-}$and $\mathrm{HO}-1^{-/-}$mice were treated with Eda and the effects on airway responsiveness to Mch were measured. The airway responsiveness of $\mathrm{Nrf} 2^{-/-}$or $\mathrm{HO}-1^{-/-}$mice to Mch was increased to a similar level as the OVA + saline group and was significantly higher compared with that of normal mice treated with Eda. This result suggested that Nrf2 or HO-1 deficiency abolished the effect of Eda on airway responsiveness to Mch. Combined with the western blot results, these findings indicated that the activated Keap1/Nrf2 pathway and HO-1 were essential in the anti-asthmatic effect of Eda.

In conclusion, Eda effectively reduced the severity of symptoms in a mouse model of asthma, which may be related to suppression of the inflammatory response and its antioxidative abilities. The activated Keap1/Nrf2 pathway and HO-1 may also be involved in the anti-asthmatic effects of Eda.

\section{Acknowledgements}

Not applicable.

\section{Funding}

The current study was funded by Shanghai Jing'an District Health Planning Commission (grant no. JWRC2014G02).

\section{Availability of data and materials}

The datasets used and/or analyzed during the current study are available from the corresponding author on reasonable request.

\section{Authors' Contributions}

$\mathrm{HC}$ designed the study, provided the funding and revised the manuscript; YP performed the airway responsiveness experiments, cell count and cytokines measurement in BALF and wrote the manuscript. WL performed the lung histopathological changes and oxidative stress experiments; YF performed the western blot analysis. JX analyzed the data.

\section{Ethical approval and consent to participate}

The present study was approved by the Institutional Animal Care and Use Committee of the Jing'an District Centre Hospital of Fudan University (approval no. AS-17-3652).

\section{Patient consent for publication}

Not applicable.

\section{Competing interests}

The authors declare that they have no competing interests.

\section{References}

1. Verhasselt V, Milcent V, Cazareth J, Kanda A, Fleury S, Dombrowicz D, Glaichenhaus N and Julia V: Breast milk-mediated transfer of an antigen induces tolerance and protection from allergic asthma. Nat Med 14: 170-175, 2008.

2. Kuipers $\mathrm{H}$ and Lambrecht BN: The interplay of dendritic cells, Th2 cells and regulatory $\mathrm{T}$ cells in asthma. Curr Opin Immunol 16: 702-708, 2004.

3. Mabalirajan U, Dinda AK, Kumar S, Roshan R, Gupta P, Sharma SK and Ghosh B: Mitochondrial structural changes and dysfunction are associated with experimental allergic asthma. J Immunol 181: 3540-3548, 2008.

4. Aguilera-Aguirre L, Bacsi A, Saavedra-Molina A, Kurosky A, Sur S and Boldogh I: Mitochondrial dysfunction increases allergic airway inflammation. J Immunol 183: 5379-5387, 2009.

5. Platts-Mills TA: The allergy epidemics: 1870-2010. J Allergy Clin Immunol 136: 3-13, 2015.

6. Li Y and Li GP: Oxidative stress in asthma: A distinct clinical and pathologic feature? J Biol Regul Homeost Agents 30: 1053-1057, 2016.

7. Mishra V, Banga $\mathbf{J}$ and Silveyra P: Oxidative stress and cellular pathways of asthma and inflammation: Therapeutic strategies and pharmacological targets. Pharmacol Ther 181: 169-182, 2018.

8. Bullone M and Lavoie JP: The contribution of oxidative stress and inflamm-aging in human and equine asthma. Int $\mathrm{J}$ Mol Sci 18: E2612, 2017.

9. Swerdlow RH: Treating neurodegeneration by modifying mitochondria: Potential solutions to a 'complex' problem. Antioxid Redox Signal 9: 1591-1603, 2007.

10. Jessie BC, Sun CQ, Irons HR, Marshall FF, Wallace DC and Petros JA: Accumulation of mitochondrial DNA deletions in the malignant prostate of patients of different ages. Exp Gerontol 37: 169-174, 2001. 
11. Hirai K, Aliev G, Nunomura A, Fujioka H, Russell RL, Atwood CS, Johnson AB, Kress Y, Vinters HV, Tabaton M, et al: Mitochondrial abnormalities in Alzheimer's disease. J Neurosci 21: 3017-3023, 2001.

12. Servais S, Boussouar A, Molnar A, Douki T, Pequignot JM and Favier R: Age-related sensitivity to lung oxidative stress during ozone exposure. Free Radic Res 39: 305-316, 2005.

13. Jang HY, Kim SM, Yuk JE, Kwon OK, Oh SR, Lee HK, Jeong H and Ahn KS: Capsicum annuum L. methanolic extract inhibits ovalbumin-induced airway inflammation and oxidative stress in a mouse model of asthma. J Med Food 14: 1144-1151, 2011.

14. Jung WK, Lee DY, Choi YH, Yea SS, Choi I, Park SG, Seo SK, Lee SW, Lee CM, Kim SK, et al: Caffeic acid phenethyl ester attenuates allergic airway inflammation and hyperresponsiveness in murine model of ovalbumin-induced asthma. Life Sci 82 797-805, 2008

15. Eisner MD, Yelin EH, Katz PP, Earnest G and Blanc PD: Exposure to indoor combustion and adult asthma outcomes: Environmental tobacco smoke, gas stoves, and woodsmoke. Thorax 57: 973-978, 2002.

16. Emelyanov A,Fedoseev G, Abulimity A, Rudinski K, Fedoulov A Karabanov A and Barnes PJ: Elevated concentrations of exhaled hydrogen peroxide in asthmatic patients. Chest 120: 1136-1139, 2001 .

17. Ganas K, Loukides S, Papatheodorou G, Panagou P and Kalogeropoulos N: Total nitrite/nitrate in expired breath condensate of patients with asthma. Respir Med 95: 649-654, 2001.

18. Kharitonov SA, Yates D, Springall DR, Buttery L, Polak J, Robbins RA and Barnes PJ: Exhaled nitric oxide is increased in asthma. Chest 107 (Suppl 3): S156-S157, 1995.

19. Noor JI,Ikeda T, Ueda Y and Ikenoue T: A free radical scavenger, edaravone, inhibits lipid peroxidation and the production of nitric oxide in hypoxic-ischemic brain damage of neonatal rats Am J Obstet Gynecol 193: 1703-1708, 2005.

20. Zhang Z, Luo Z, Bi A, Yang W, An W, Dong X, Chen R, Yang S, Tang H, Han X and Luo L: Compound edaravone alleviates lipopolysaccharide (LPS)-induced acute lung injury in mice. Eur J Pharmacol 811: 1-11, 2017.

21. Han CH, Guan ZB, Zhang PX, Fang HL, Li L, Zhang HM, Zhou FJ, Mao YF and Liu WW: Oxidative stress induced necroptosis activation is involved in the pathogenesis of hyperoxic acute lung injury. Biochem Biophys Res Commun 495: 2178-2183, 2018.

22. Wang X, Lai R, Su X, Chen G, Liang Z: Edaravone attenuates lipopolysaccharide-induced acute respiratory distress syndrome associated early pulmonary fibrosis via amelioration of oxidative stress and transforming growth factor- $\beta 1 / \mathrm{Smad} 3$ signaling. Biochem Biophys Res Commun 495: 706-712, 2018.

23. Uchiyama M, Tojo K, Yazawa T, Ota S, Goto T and Kurahashi K Edaravone prevents lung injury induced by hepatic ischemia-reperfusion. J Surg Res 194: 551-557, 2015

24. Liu S, Liu K, Sun Q, Liu W, Xu W, Denoble P, Tao H and Sun X: Consumption of hydrogen water reduces paraquat-induced acute lung injury in rats. J Biomed Biotechnol 2011: 305086, 2011.

25. Cheng ZQ, Han JY, Sun P, Weng YY, Chen J, Wu GY and Ma HX: Edaravone attenuates paraquat-induced lung injury by inhibiting oxidative stress in human type II alveolar epithelial cells. World J Emerg Med 3: 55-59, 2012

26. Xu JZ, Shen BZ, Li Y, Zhang T, Xu WH, Liu XW and Lu HG: Edaravone attenuates ischemia-reperfusion injury by inhibiting oxidative stress in a canine lung transplantation model. Chin Med J (Engl) 121: 1583-1587, 2008

27. Keum YS, Owuor ED, Kim BR, Hu R and Kong AN: Involvement of Nrf2 and JNK1 in the activation of antioxidant responsive element (ARE) by chemopreventive agent phenethyl isothiocyanate (PEITC). Pharm Res 20: 1351-1356, 2003.

28. Zhang J, Shi X, Chen Z, Geng J, Wang Y, Feng H, Zhu G and Chen Q: Edaravone reduces iron-mediated hydrocephalus and behavioral disorder in rat by activating the $\mathrm{Nrf} 2 / \mathrm{HO}-1$ pathway. J Stroke Cerebrovasc Dis 27: 3511-3520, 2018.

29. Zhang D, Xiao Y, Lv P, Teng Z, Dong Y, Qi Q and Liu Z: Edaravone attenuates oxidative stress induced by chronic cerebral hypoperfusion injury: Role of ERK/Nrf2/HO-1 signaling pathway. Neurol Res 40: 1-10, 2018

30. Liu Z, Yang C, Meng X, Li Z, Lv C and Cao P: Neuroprotection of edaravone on the hippocampus of kainate-induced epilepsy rats through Nrf2/HO-1 pathway. Neurochem Int 112: 159-165, 2018 .
31. Ogata-Suetsugu S, Yanagihara T, Hamada N, Ikeda-Harada C, Yokoyama T, Suzuki K, Kawaguchi T, Maeyama T, Kuwano K and Nakanishi Y: Amphiregulin suppresses epithelial cell apoptosis in lipopolysaccharide-induced lung injury in mice. Biochem Biophys Res Commun 484: 422-428, 2017.

32. Feng J, Li P, Li X and Zhou H: Anatomic Distribution and morphology of common tracheal/bronchial/pulmonary cells. In: Rapid On-Site Evaluation (ROSE) in Diagnostic Interventional Pulmonology. Feng J, Li X, Li P, Li Q and Shi Y (eds). Springer, Singapore, 2019

33. Guedes AG, Jude JA, Paulin J, Rivero-Nava L, Kita H, Lund FE and Kannan MS: Airway responsiveness in CD38-deficient mice in allergic airway disease: Studies with bone marrow chimeras. Am J Physiol Lung Cell Mol Physiol 308: L485-L493, 2015.

34. Yin N, Peng Z, Li B, Xia J, Wang Z, Yuan J, Fang L and Lu X: Isoflurane attenuates lipopolysaccharide-induced acute lung injury by inhibiting ROS-mediated NLRP3 inflammasome activation. Am J Transl Res 8: 2033-2046, 2016.

35. Zhao P, Zhou WC, Li DL, Mo XT, Xu L, Li LC, Cui WH and Gao J: Total glucosides of Danggui Buxue Tang attenuate BLM-induced pulmonary fibrosis via regulating oxidative stress by inhibiting NOX4. Oxid Med Cell Longev 2015: 645814, 2015.

36. Barnes PJ, Ito $\mathrm{K}$ and Adcock IM: Corticosteroid resistance in chronic obstructive pulmonary disease: Inactivation of histone deacetylase. Lancet 363: 731-733, 2004.

37. Bousquet J, Jeffery PK, Busse WW, Johnson M and Vignola AM: Asthma. From bronchoconstriction to airways inflammation and remodeling. Am J Respir Crit Care Med 161: 1720-1745, 2000.

38. Frossi B, De Carli M, Piemonte M and Pucillo C: Oxidative microenvironment exerts an opposite regulatory effect on cytokine production by Th1 and Th2 cells. Mol Immunol 45: 58-64, 2008.

39. Yang T, Zhang J, Sun L, Zhu X, Li J, Wang J, Chen H, Bao R, Deng X, Hou J and Liu Y: Combined effects of a neutrophil elastase inhibitor (sivelestat sodium) and a free radical scavenger (edaravone) on lipopolysaccharide-induced acute lung injury in rats. Inflamm Res 61: 563-569, 2012.

40. Wang Z, Li R, Liu Y, Liu X, Chen W, Xu S, Guo Y, Duan J, Chen $\mathrm{Y}$ and Wang C: Protective effects of edaravone combined puerarin on inhalation lung injury induced by black gunpowder smog. Int Immunopharmacol 26: 125-132, 2015.

41. Zhang W, Guo Y, Yu S, Wei J and Jin J: Effects of edaravone on the expression of $\beta$-defensin- 2 mRNA in lung tissue of rats with myocardial ischemia reperfusion. Mol Med Rep 7: 1683-1687, 2013.

42. Sugiura $\mathrm{H}$ and Ichinose $\mathrm{M}$ : Oxidative and nitrative stress in bronchial asthma. Antioxid Redox Signal 10: 785-797, 2008.

43. Hoshino T, Okamoto M, Takei S, Sakazaki Y, Iwanaga T and Aizawa H: Redox-regulated mechanisms in asthma. Antioxid Redox Signal 10: 769-783, 2008.

44. Castillo SS, Levy M, Thaikoottathil JV and Goldkorn T: Reactive nitrogen and oxygen species activate different sphingomyelinases to induce apoptosis in airway epithelial cells. Exp Cell Res 313: 2680-2686, 2007

45. Katsumata U, Miura M, Ichinose M, Kimura K, Takahashi T, Inoue $\mathrm{H}$ and Takishima $\mathrm{T}$ : Oxygen radicals produce airway constriction and hyperresponsiveness in anesthetized cats. Am Rev Respir Dis 141: 1158-1161, 1990.

46. King MR, Ismail AS, Davis LS and Karp DR: Oxidative stress promotes polarization of human $\mathrm{T}$ cell differentiation toward a $\mathrm{T}$ helper 2 phenotype. J Immunol 176: 2765-2772, 2006.

47. Imaoka H, Hoshino T, Takei S, Sakazaki Y, Kinoshita T, Okamoto M, Kawayama T, Yodoi J, Kato S, Iwanaga T and Aizawa H: Effects of thioredoxin on established airway remodeling in a chronic antigen exposure asthma model. Biochem Biophys Res Commun 360: 525-530, 2007.

48. Mitsunobu F, Yamaoka K, Hanamoto K, Kojima S, Hosaki Y, Ashida K, Sugita K and Tanizaki Y: Elevation of antioxidant enzymes in the clinical effects of radon and thermal therapy for bronchial asthma. J Radiat Res 44: 95-99, 2003.

49. Bucchieri F, Puddicombe SM, Lordan JL, Richter A, Buchanan D, Wilson SJ, Ward J, Zummo G, Howarth PH, Djukanović R, et al: Asthmatic bronchial epithelium is more susceptible to oxidant-induced apoptosis. Am J Respir Cell Mol Biol 27: 179-185, 2002.

50. Crapo JD: Oxidative stress as an initiator of cytokine release and cell damage. Eur Respir J Suppl 44: S4-S6, 2003.

51. Boljevic S, Daniljak IG and Kogan AH: Changes in free radicals and possibility of their correction in patients with bronchial asthma. Vojnosanit Pregl 50: 3-18, 1993 (In Russian, Serbian). 
52. Fang WT, Li HJ, Zhou LS: Protective effects of prostaglandin E1 on human umbilical vein endothelial cell injury induced by hydrogen peroxide. Acta Pharmacol Sin 31: 485-492, 2010.

53. Yamamoto H, Yamamoto Y, Yamagami K, Kume M, Kimoto S, Toyokuni S, Uchida K, Fukumoto M and Yamaoka Y: Heat-shock preconditioning reduces oxidative protein denaturation and ameliorates liver injury by carbon tetrachloride in rats. Res Exp Med (Berl) 199: 309-318, 2000.

54. Zhao X, Jin L, Shen N, Xu B, Zhang W, Zhu H and Luo Z: Salidroside inhibits endogenous hydrogen peroxide induced cytotoxicity of endothelial cells. Biol Pharm Bull 36: 1773-1778, 2013.

55. Zhao SF, Zhang QR, Xia SJ, Lin SR and Gu F: Influence of Yiqi Dingchuan decoction on antioxidant ability of alvoelar macrophages in Guinea pigs with asthma. Chin J Basic Med Tradit Chin Med 6: 31-34, 2000.

56. Mach WJ, Thimmesch AR, Pierce JT and Pierce JD: Consequences of hyperoxia and the toxicity of oxygen in the lung. Nurs Res Pract 2011: 260482, 2011.
57. Li Y, Liu H, Zeng W and Wei J: Edaravone protects against hyperosmolarity-induced oxidative stress and apoptosis in primary human corneal epithelial cells. PLoS One 12: e0174437, 2017.

58. Jangra A, Kwatra M, Singh T, Pant R, Kushwah P, Ahmed S, Dwivedi D, Saroha B and Lahkar M: Edaravone alleviates cisplatin-induced neurobehavioral deficits via modulation of oxidative stress and inflammatory mediators in the rat hippocampus. Eur J Pharmacol 791: 51-61, 2016.

59. Li AL, Shen T, Wang T, Zhou MX, Wang B, Song JT, Zhang PL, Wang XL, Ren DM, Lou HX and Wang XN: Novel diterpenoid-type activators of the Keap1/Nrf2/ARE signaling pathway and their regulation of redox homeostasis. Free Radic Biol Med 141: 21-33, 2019.

60. Kobayashi M and Yamamoto M: Molecular mechanisms activating the Nrf2-Keap1 pathway of antioxidant gene regulation. Antioxid Redox Signal 7: 385-394, 2005. 